\title{
Simplified Model for Light Propagation in Graded-Index-Medium
}

\author{
Rabi Ibrahim Rabady \\ Electrical Engineering Department, Jordan University of Science and Technology, Irbid, Jordan \\ Email: rrabady@just.edu.jo,rabirabady@yahoo.com
}

Received March 2, 2013; revised April 5, 2013; accepted May 3, 2013

Copyright (C) 2013 Rabi Ibrahim Rabady. This is an open access article distributed under the Creative Commons Attribution License, which permits unrestricted use, distribution, and reproduction in any medium, provided the original work is properly cited.

\begin{abstract}
Using the ray theory of light, a simple theoretical model for the power evolution of a propagating light in graded-index-medium is presented. This work can be useful for different engineering applications that utilize graded-index-material, and for further understanding of natural phenomena that depends on light propagation in graded-index-medium.
\end{abstract}

Keywords: Graded-Index Medium; Fresnel Reflection; Power Evolution

\section{Introduction}

Light propagation in graded-index-medium is found in different natural processes and engineering applications, such as mirage phenomenon, wavelength multiplexing and demultiplexing, graded-index fibers and graded-index lenses [1-3].

Generally, as light propagates in graded-index-material at large scale, it encounters two major effects; namely, reflection and refraction. This work utilizes the simple laws of reflection and refraction to present a useful insight of the light propagation in graded-index-medium by accounting the gradual Fresnel reflection and refraction. Surely, using Maxwell equations leads to a more comprehensive and accurate solution; however, this requires a much complicated modeling and simulation because of the spatial dependency of the medium refractive-index. Therefore, a simple model will be developed in order to quantify the power evolution of propagating light in a defined graded-index medium.

\section{Theory}

When light propagates in graded-index-medium it experiences gradual refraction and reflection simultaneously. The gradual refraction behavior is modeled using both the ray theory [4-6] and the wave theory $[7,8]$ of light. However, a simple modeling of the light gradual reflection in graded-index-medium can be achieved by considering a propagating ray of light through a gradedindex-medium that changes its refractive index in the y-direction, as depicted in Figure 1. Therefore, as light enters a sliced layer of the medium it travels through that layer distance $\Delta s$, also the incident angle changes from $\theta_{1}$ to $\theta_{2}$, whereas, the refractive index changes from $n(y)=$ $n_{1}$ to $n(y+\Delta y)=n_{2}$. Where, $\theta_{1}$ and $\theta_{2}$ are the incident and transmitted ray angles with respect to norm of the sliced layer, respectively. If light enters the graded index medium with incident angle other than the normal incidence, it would bend gradually in order to satisfy Snell's law. Moreover, light will experience spatial dispersion and power reduction at each point on the ray path trajectory as shown in Figure 1; where, $\mathrm{d} P$ is the reflected light power because of gradual Fresnel reflection that stems from gradual refractive index change through the graded-index-medium. Therefore, the Fresnel reflection coefficient of electric and magnetic polarized light can be expressed as [7]:

$$
\begin{array}{r}
r_{E}=\frac{E_{\perp}^{r}}{E_{\perp}^{i}}=-\frac{\sin \left(\theta_{1}-\theta_{2}\right)}{\sin \left(\theta_{1}+\theta_{2}\right)}=\frac{\sin \theta_{2} \cos \theta_{1}-\cos \theta_{2} \sin \theta_{1}}{\sin \theta_{1} \cos \theta_{2}+\sin \theta_{2} \cos \theta_{1}} \\
r_{H}=\frac{E_{\|}^{r}}{E_{\|}^{i}}=\frac{\tan \left(\theta_{1}-\theta_{2}\right)}{\tan \left(\theta_{1}+\theta_{2}\right)}
\end{array}
$$

Equation (2) can be expressed in terms of angles' tangents as:

$$
r_{H}=\left(\frac{\tan \theta_{1}-\tan \theta_{2}}{1+\tan \theta_{1} \tan \theta_{2}}\right)\left(\frac{1-\tan \theta_{1} \tan \theta_{2}}{\tan \theta_{1}+\tan \theta_{2}}\right)
$$

where $E_{\perp}^{i}$ and $E_{\perp}^{r}$ are the incident and reflected elec- 


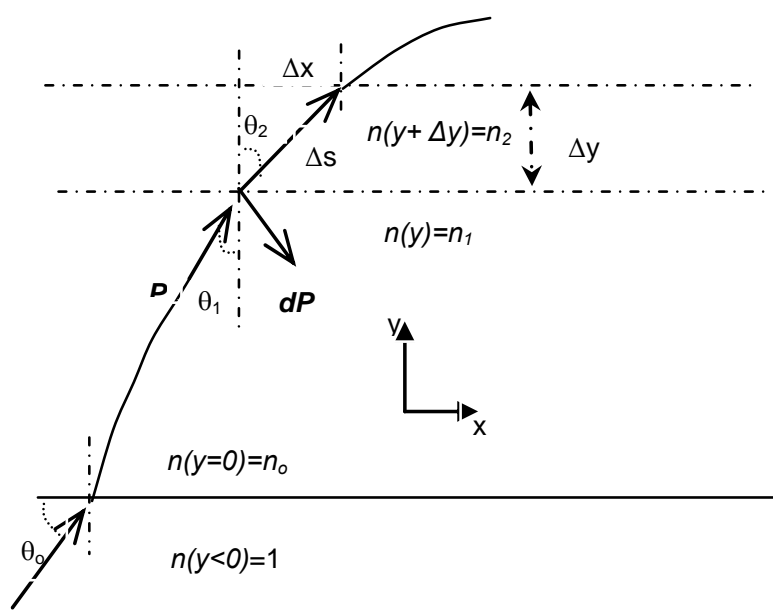

Figure 1. Ray optics of light propagation in graded-refractive-index-medium.

tric fields for the electric polarized light; whereas, $E_{\|}^{i}$ and $E_{\|}^{r}$ are the incident and reflected electric fields for the magnetic polarized light, respectively.

The reflected power $\mathrm{d} P$, which is cut from the incident power $P$, to the incident power ratio can be found for the electric polarized light by:

$$
\left(\frac{\mathrm{d} P}{P}\right)_{E}=\left|\frac{E_{\perp}^{r}}{E_{\perp}^{i}}\right|^{2}=\left(\frac{\sin \theta_{2} \cos \theta_{1}-\cos \theta_{2} \sin \theta_{1}}{\sin \theta_{2} \cos \theta_{1}+\cos \theta_{2} \sin \theta_{1}}\right)^{2}
$$

Dividing all terms in the numerator and the dominator by $\cos \theta_{2} \cos \theta_{1}$, Equation (4) becomes:

$$
\left(\frac{\mathrm{d} P}{P}\right)_{E}=\left(\frac{\tan \theta_{2}-\tan \theta_{1}}{\tan \theta_{2}+\tan \theta_{1}}\right)^{2}
$$

Moreover, using Equation (3) a similar equation for the reflected power " $\mathrm{d} P$ " to the incident power " $P$ " ratio can be obtained for the magnetic polarized light:

$$
\left(\frac{\mathrm{d} P}{P}\right)_{H}=\left|\frac{E_{\|}^{r}}{E_{\|}^{i}}\right|^{2}=\left[\left(\frac{\tan \theta_{1}-\tan \theta_{2}}{1+\tan \theta_{1} \tan \theta_{2}}\right)\left(\frac{1-\tan \theta_{1} \tan \theta_{2}}{\tan \theta_{1}+\tan \theta_{2}}\right)\right]^{2}
$$

Given $y(x)$ is the path trajectory of the propagating light in the graded-index-medium, as shown in Figure 1, the tangent of the incident ray angle could be expressed by:

$$
\tan \theta_{1}=\left(\frac{\mathrm{d} y}{\mathrm{~d} x}\right)^{-1}=\frac{1}{y^{\prime}(x)}
$$

Moreover, using the following relation:

$$
\frac{\mathrm{d}^{2} y}{\mathrm{~d} x^{2}}=y^{\prime \prime}(x)=\frac{y^{\prime}(x+\mathrm{d} x)-y^{\prime}(x)}{\mathrm{d} x}
$$

which can be rearranged into:

$$
y^{\prime}(x+d x)=y^{\prime \prime}(x) \mathrm{d} x+y^{\prime}(x)
$$

Therefore, the tangent of the transmittance angle $\theta_{2}$ becomes

$$
\tan \theta_{2}=\frac{1}{y^{\prime \prime}(x) \mathrm{d} x+y^{\prime}(x)}
$$

Substituting Equations (7) and (8) in Equations (5) and (6) yields:

$$
\begin{gathered}
\left(\frac{\mathrm{d} P}{P}\right)_{E}=\left(\frac{y^{\prime \prime} \mathrm{d} x}{2 y^{\prime}+y^{\prime \prime} \mathrm{d} x}\right)^{2} \\
\left(\frac{\mathrm{d} P}{P}\right)_{H}=\left[\left(\frac{y^{\prime \prime} \mathrm{d} x}{2 y^{\prime}+y^{\prime \prime} \mathrm{d} x}\right)\left(\frac{1-y^{\prime 2}-y^{\prime} y^{\prime \prime} \mathrm{d} x}{1+y^{\prime 2}+y^{\prime} y^{\prime \prime} \mathrm{d} x}\right)\right]^{2}
\end{gathered}
$$

Equations (9) and (10) represent nonlinear differential equations that permits predicting the power evolution as light propagate in graded-index-medium for both polarizations. In order to solve Equations (9) and (10), the path trajectory $y(x)$ of light inside the graded-index-medium need to be found since it decides the right hand side of Equations (9) and (10).

Obviously, $y^{\prime}$ and $y^{\prime \prime}$ need to be found before determining the right hand side of Equations (9) and (10). In order to achieve this goal, we start with the eikonal equation [7]:

$$
\frac{\mathrm{d}}{\mathrm{d} s}\left(n(\boldsymbol{r}) \frac{\mathrm{d} r}{\mathrm{~d} s}\right)=\nabla n(\boldsymbol{r})
$$

where, $s$ is the distance along the ray path, and $r$ is the position vector of any point on the ray path.

Since the refractive index changes with respect to the $\mathrm{y}$-axis only, Equation (11) reduces to:

$$
\frac{\mathrm{d}}{\mathrm{d} s}\left(n(y) \frac{\mathrm{d} y}{\mathrm{~d} s}\right)=\frac{\mathrm{d} n(y)}{\mathrm{d} y}
$$

$$
\text { Moreover, substituting } \mathrm{d} s=\frac{\mathrm{d} x}{\sin \theta} \text { in Equation }
$$
yields:

$$
\sin ^{2} \theta \frac{\mathrm{d}}{\mathrm{d} x}\left(n(y) \frac{\mathrm{d} y}{\mathrm{~d} x}\right)=\frac{\mathrm{d} n(y)}{\mathrm{d} y}
$$

Since the refractive index is changing only in the $y$-direction, Equation (13) becomes:

$$
n(y) \sin ^{2} \theta\left(\frac{\mathrm{d}^{2} y}{\mathrm{~d} x^{2}}\right)=\frac{\mathrm{d} n(y)}{\mathrm{d} y}
$$

Introducing the ray invariant $\beta$, which is defined as:

$$
\beta=n(y) \sin \theta(y)=n(0) \sin \theta(0)
$$

where $n(0)$ and $\theta(0)$ are the refractive index and the angle of the ray with respect to $x$-axis at the entrance of the graded-index-medium, respectively. Whereas, $n(y)$ is the refractive index) and $\theta(y)$ is the angle between the ray 
and the $x$-axis.

Substituting Equation (15) into Equation (14) yields:

$$
2 \beta^{2} \frac{\mathrm{d}^{2} y}{\mathrm{~d} x^{2}}=\frac{\mathrm{d} n^{2}(y)}{\mathrm{d} y}
$$

Thus,

$$
y^{\prime \prime}=\frac{\mathrm{d}^{2} y}{\mathrm{~d} x^{2}}=\frac{n(y) \frac{\mathrm{d} n(y)}{\mathrm{d} y}}{\beta^{2}}
$$

Moreover, equation 15 can be written as:

$$
\sin \theta(y)=\frac{\beta}{n(y)}
$$

Therefore,

$$
y^{\prime}=\frac{\mathrm{d} y}{\mathrm{~d} x}=\frac{1}{\tan \theta}=\frac{\sqrt{n^{2}(y)-\beta^{2}}}{\beta}
$$

Given that $n(0), \theta(0), P(0)$, and $n(y)$ are all defined, it would be straight forward to solve Equations (9) and (10) numerically after substituting Equations (17) and (18); hence, it would be possible to predict the power evolu0 tion of light as it propagate inside a graded-index-me0 dium for electric and magnetic polarized light.

It would be useful to mention here that similarly the same results can be used for the slanted incident rays; the only difference is to consider a new $x$-axis that comes from the projection of the slanted ray on interface surface between the homogeneous and graded-index-medium.

\section{Simulation and Results}

Equations (9) and (10) can be solved numerically by writing them as difference equations using the following iterative form:

$$
P\left(y_{i+1}\right)=P\left(y_{i}\right)\left(1-R H\left(y_{i}\right)\right)
$$

where $R H\left(y_{i}\right)$ is the right hand side of Equations (9) and (10), and " $i$ " is the iterative running index; whereas, $y_{i}$, and $\Delta x_{i}$ are obtained by:

$$
\begin{aligned}
& y_{i+1}=y_{i}+\Delta y \\
& \Delta x_{i}=\frac{\Delta y}{\left.\frac{\mathrm{d} y}{\mathrm{~d} x}\right|_{y_{i}}}
\end{aligned}
$$

$n\left(y_{i}\right)$ is obtained by sampling $n(y)$ every $\Delta y$, therefore, the derivative $\frac{\mathrm{d} n(y)}{\mathrm{d} y}$ is found numerically by:

$$
\left.\frac{\mathrm{d} n(y)}{\mathrm{d} y}\right|_{y_{i}}=\frac{n\left(y_{i+1}\right)-n\left(y_{i}\right)}{\Delta y}
$$

For a given refractive index profile $n\left(y_{i}\right)$, Equations
(20) and (21) are substituted in Equations (17) and (18) to obtain $y_{i}^{\prime}$ and $y_{i}^{\prime \prime}$ numerically, respectively. Consequently, $R H\left(y_{i}\right)$ in Equation (19) can also be determined numerically from Equations (9) and (10).

In the following we consider a numerical example in order to quantify the power evolution as light propagates in a graded-index-medium. For the sake of simplicity we consider a linearly decreasing refractive index profile for the graded-index-medium such that $n(y)=n_{o}-s y$, where, $n_{o}$ is the refractive index at the entrance of the gradedindex-medium $(y=x=0)$, and $s$ represents the gradient of the refractive index in the $y$-direction as assumed in the theory section.

Figure 2 shows the evolution of the normalized power with respect to the power of the entering light power $P(0)$ in $\mathrm{dB}$ versus the horizontal distance $x$ with the following parameters: $\theta_{o}=\pi / 4, n_{o}=2$, and $s=0.01 \mathrm{~mm}^{-1}$. Obviously, the magnetic polarization curve comes above the electric polarization curve; this is attributed to the fact that the Fresnel reflection of the electric polarization is greater than the Fresnel reflection of the magnetic polarization at all incident angles. The knee shape in the magnetic polarization curve is due to the light passing through theBrewster angle as it bends inward in the decreasing-refractive-index-medium. The turning point, which is the point where light experience total internal reflection, was found to be at $(x=117.05 \mathrm{~mm}, y=$ $129.29 \mathrm{~mm}$ ). It is important to emphasize here that the gradual reflection of light while propagating through a decreasing-refractive-index-medium not only reduces the propagating power, but also leads to a spatial dispersion of light because the reflected portions of light follow different paths from the path of the main ray. Figure 3 is a zoom on of Figure 2 for the last two millimeters along

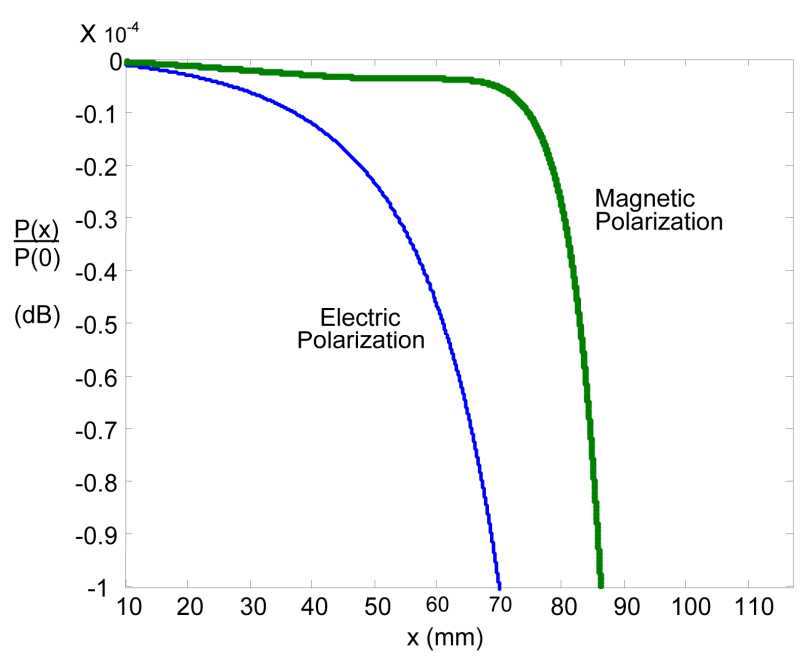

Figure 2. Evolution of the normalized power, in $\mathrm{dB}$, as light propagates in a linearly decreasing graded-index medium in the $y$-direction versus the horizontal distance $x\left(\theta_{0}=\pi / 4\right.$, no $=2$, and $s=0.01 \mathrm{~mm}^{-1}$ ). 


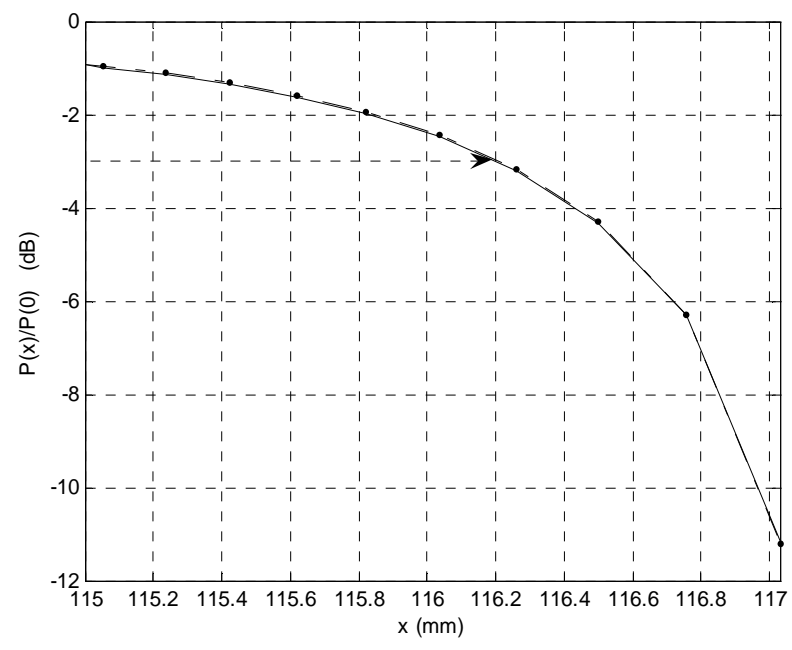

Figure 3. Zoom on the last two millimeters of Figure 2 before the light total internal reflection.

the $x$-direction before light total internal reflection, which also shows that only half of the power is contained within the last $0.8 \mathrm{~mm}$. This effect is critical since it decides the devise spectral/spatial resolution when gradedindex material is used for wavelength multiplexing and demultiplexing.

\section{Conclusion}

In this paper, the power evolution of a propagating light inside a graded-refractive-index-medium was modeled and numerically simulated for both the electric and the magnetic polarizations using the simple ray theory of light. Therefore, it would be possible to predict the light intensity in the graded-index-medium. Moreover, it was shown that the spatial dispersion and the associated power reduction of the light main ray are more pronounced for the electric polarized light than that of the magnetic polarized light; this can be attributed to the fact that the angular Fresnel reflection for the electric polarized light is greater at any incident angles. This work can be useful for the different engineering applications that utilize graded-index-medium; further more, it could provide more insight of natural phenomena that depend on the light propagation in graded-index-medium.

\section{REFERENCES}

[1] M. Heckler and A. Dreher, "Analysis of Graded-Index Fibers Using a Full-Wave Equivalent Circuit," Journal of Lightwave Technology, Vol. 25, No. 1, 2007, pp. 346-349. http://dx.doi.org/10.1109/JLT.2006.886676

[2] H. B. Salameh and M. Irshid, "Wavelength-Division Demultiplexing Using Graded-Index Planar Structures," Journal of Lightwave Technology, Vol. 24, No. 6, 2006, pp. 2401-2408. http://dx.doi.org/10.1109/JLT.2006.874589

[3] B. Allman, A. Klein, K. Nugent and G. Opat, "Refractive-Index-Profile Determination by Using Lloyd's Mirage," Applied Optics, Vol. 33, No. 10, 1994, pp. 18061811. http://dx.doi.org/10.1364/AO.33.001806

[4] D. Bertilone and C. Pask, "Exact Ray Paths in a GradedIndex Taper," Applied Optics, Vol. 26, No. 7, 1987, pp. 1189-1194.

[5] E. W. Marchand, "Graded Index Optics," Academic, New York, 1978.

[6] A. W. Snyder and J. D. Love, "Optical Waveguide Theory," Chapman \& Hall, New York, 1983.

[7] M. Born and E. Wolf, "Principles of Optics," 7th Edition, Cambridge University, Cambridge, 1999.

[8] R. Pratesi and L. Ronchi, "Wave Propagation in a Nonparabolic Graded-Index Medium," IEEE Transactions on Microwave Theory and Techniques, Vol. 26, No. 11, 1978 , pp. 856-858. 